\title{
AMENDMENTS
}

\section{Author Correction: Biallelic expansion of an intronic repeat in RFC1 is a common cause of late-onset ataxia}

Andrea Cortese (1), Roberto Simone (D), Roisin Sullivan, Jana Vandrovcova, Huma Tariq, Wai Yan Yau, Jack Humphrey (i), Zane Jaunmuktane, Prasanth Sivakumar, James Polke, Muhammad Ilyas, Eloise Tribollet, Pedro J. Tomaselli, Grazia Devigili, Ilaria Callegari, Maurizio Versino, Vincenzo Salpietro, Stephanie Efthymiou (1, Diego Kaski, Nick W. Wood, Nadja S. Andrade, Elena Buglo, Adriana Rebelo, Alexander M. Rossor, Adolfo Bronstein, Pietro Fratta, Wilson J. Marques, Stephan Züchner, Mary M. Reilly and Henry Houlden (1)

Correction to: Nature Genetics https://doi.org/10.1038/s41588-019-0372-4, published online 29 March 2019.

In the version of this article initially published, the name of author Wai Yan Yau was misspelled. The error has been corrected in the HTML and PDF versions of the article.

\section{Author Correction: An atlas of genetic influences on osteoporosis in humans and mice}

John A. Morris (1), John P. Kemp (D), Scott E. Youlten (D), Laetitia Laurent, John G. Logan, Ryan C. Chai, Nicholas A. Vulpescu (1), Vincenzo Forgetta, Aaron Kleinman, Sindhu T. Mohanty, C. Marcelo Sergio (D, Julian Quinn, Loan Nguyen-Yamamoto, Aimee-Lee Luco, Jinchu Vijay, Marie-Michelle Simon, Albena Pramatarova, Carolina Medina-Gomez @ , Katerina Trajanoska, Elena J. Ghirardello, Natalie C. Butterfield, Katharine F. Curry, Victoria D. Leitch, Penny C. Sparkes, Anne-Tounsia Adoum, Naila S. Mannan, Davide S. K. Komla-Ebri, Andrea S. Pollard, Hannah F. Dewhurst, Thomas A. D. Hassall, Michael-John G. Beltejar, 23andMe Research Team, Douglas J. Adams, Suzanne M. Vaillancourt, Stephen Kaptoge, Paul Baldock, Cyrus Cooper, Jonathan Reeve (1), Evangelia E. Ntzani $\mathbb{D}^{\mathbb{D}}$, Evangelos Evangelou, Claes Ohlsson, David Karasik, Fernando Rivadeneira (D), Douglas P. Kiel(D), Jonathan H. Tobias, Celia L. Gregson (1), Nicholas C. Harvey (1D), Elin Grundberg, David Goltzman, David J. Adams, Christopher J. Lelliott (®), David A. Hinds @D, Cheryl L. Ackert-Bicknell, Yi-Hsiang Hsu, Matthew T. Maurano, Peter I. Croucher, Graham R. Williams (D, J. H. Duncan Bassett (D), David M. Evans (D) and J. Brent Richards (D)

Correction to: Nature Genetics https://doi.org/10.1038/s41588-018-0302-x, published online 31 December 2018.

In the version of this article initially published, in Fig. 5a, the data in the right column of 'DAAM2 gRNA1' were incorrectly plotted as circles indicating 'untreated' rather than as squares indicating 'treated'. The error has been corrected in the HTML and PDF versions of the article.

\section{Author Correction: Transposable elements drive widespread expression of oncogenes in human cancers}

Hyo Sik Jang (D, Nakul M. Shah, Alan Y. Du, Zea Z. Dailey, Erica C. Pehrsson, Paula M. Godoy, David Zhang, Daofeng Li, Xiaoyun Xing, Sungsu Kim, David O'Donnell, Jeffrey I. Gordon and Ting Wang (i)

Correction to: Nature Genetics https://doi.org/10.1038/s41588-019-0373-3, published online 29 March 2019.

In the version of this article initially published, grant PF-17-201-01-TBG from the American Cancer Society to author Erica C. Pehrsson was not included in the Acknowledgements. The error has been corrected in the HTML and PDF versions of the article. 